ICBT FOR PERFECTIONISM

\title{
A Randomized Controlled Trial of Internet-Based Cognitive Behavior Therapy for Perfectionism Including an Investigation of Outcome Predictors
}

Alexander Rozental ${ }^{1 *}$, MSc; Roz Shafran ${ }^{2}, \mathrm{PhD}$; Tracey Wade ${ }^{3}, \mathrm{PhD} ; \mathrm{Sarah} \mathrm{Egan}^{4}, \mathrm{PhD}$; Lise Bergman Nordgren ${ }^{5}$, PhD; Per Carlbring ${ }^{1}, \mathrm{PhD}$; Andreas Landström ${ }^{5}$, MSc; Stina Roos ${ }^{5}$, MSc; Malin Skoglund ${ }^{5}$, MSc; Elisabet Thelander ${ }^{5}, \mathrm{MSc}_{\text {; }}$ Linnéa Trosell ${ }^{5}, \mathrm{MSc}$; Alexander Örtenholm ${ }^{5}$, MSc; Gerhard Andersson ${ }^{5,6}, \mathrm{PhD}$

1 Department of Psychology, Stockholm University, Stockholm, Sweden 2 Institute of Child Health, University College London, London, England 3 School of Psychology, Flinders University, Adelaide, Australia 4 School of Psychology and Speech Pathology, Curtin University, Perth, Australia 5 Department of Behavioural Sciences and Learning, Linköping University, Linköping, Sweden

6 Department of Clinical Neuroscience, Karolinska Institutet, Stockholm, Sweden

* Corresponding author: Alexander Rozental, MSc. Division of Clinical Psychology, Department of Psychology, Stockholm University, Frescati Hagväg 8, Stockholm, 10691 Phone: 468163871 Fax: 468159342 Email: alexander.rozental@ psychology.su.se 


\title{
ICBT FOR PERFECTIONISM
}

\begin{abstract}
Being highly attentive to details can be a positive feature. However, for some individuals, perfectionism can lead to distress and is associated with many psychiatric disorders.

Cognitive behavior therapy has been shown to yield many benefits for those experiencing problems with perfectionism, but the access to evidence-based care is limited. The current study investigated the efficacy of guided Internet-based Cognitive Behavior Therapy (ICBT) and predictors of treatment outcome. In total, 156 individuals were included and randomized to an eight-week treatment or wait-list control. Self-report measures of perfectionism, depression, anxiety, self-criticism, self-compassion, and quality of life were distributed during screening and at post-treatment. Intention-to-treat were used for all statistical analyses. Moderate to large between-group effect sizes were obtained for the primary outcome measures, Frost Multidimensional Perfectionism Scale, subscales Concerns over Mistakes and Personal Standards, Cohen's $d=0.68-1.00,95 \%$ Confidence Interval (CI) [0.36-1.33], with $35(44.9 \%)$ of the patients in treatment being improved. Predictors were also explored, but none were related to treatment outcome. In sum, guided ICBT can be helpful for addressing problems with clinical perfectionism, but research of its long-term benefits is warranted.
\end{abstract}

Keywords: Internet-based cognitive behavior therapy, perfectionism, predictors. 
ICBT FOR PERFECTIONISM

\section{Highlights}

- The treatment yielded large between-group effect sizes for clinical perfectionism.

- Moderate between-group effect sizes were obtained for depression, anxiety, and quality of life.

- Adherence was high, $63(80.8 \%)$ completed $\geq 75 \%$ of the modules in eight weeks. 


\section{ICBT FOR PERFECTIONISM}

\section{Introduction}

Perfectionism describes the characteristics of an individual who sets high standards and strives "compulsively and unremittingly" towards achievement (Burns, 1980, p. 34). Albeit often regarded as desirable, perfectionism can also involve a set of assumptions and behaviors that are maladaptive (Stumpf \& Parker, 2000). Stoeber and Otto (2006) differentiate between helpful and unhelpful perfectionism, where the latter is related to excessive worry, avoidance, procrastination, and self-criticism. Unhelpful, or, clinical perfectionism, is also assumed to be associated with stress and interpersonal difficulties (Chang, Watkins, \& Banks, 2004), and contributing to many psychiatric disorders, such as, obsessive-compulsive disorder, major depressive disorder, and eating disorders (Shafran \& Mansell, 2001). As such, Egan, Wade, and Shafran (2011) refer to perfectionism as a transdiagnostic issue as well as a feature that can impede treatment progress for psychiatric disorders. Clinical perfectionism was defined by Shafran, Cooper, and Fairburn (2002) as "the overdependence of self-evaluation on the determined pursuit of personally demanding, self-imposed, standards in at least one highly salient domain, despite adverse consequences" (p. 778). From this perspective, perfectionism becomes problematic if it is characterized by highly rigid standards, which the individual then strives to accomplish because failure has negative consequences for self-evaluation, e.g., being perceived as inadequate. Clinical perfectionism is related to cognitive biases, such as discounting the positive, as well as emotional arousal. It is also linked to counter-productive behaviors, for example, repetitive checking, and avoidance of specific tasks or activities (Shafran et al., 2002), thereby being maintained and often leading to distress.

Cognitive Behavior Therapy (CBT) is regarded as a promising treatment for clinical perfectionism, but few clinical trials have been performed. Notable exceptions do exist, such as, CBT being delivered individually face-to-face (Egan \& Hine, 2008), CBT administered in groups (Handley, Egan, Kane, \& Rees, 2015), guided self-help (Steele \& Wade, 2008), and 


\section{ICBT FOR PERFECTIONISM}

unguided self-help (Radhu, Daskalakis, Arpin-Cribbie, Irvine, \& Ritvo, 2012). Lloyd, Schmidt, Khondoker, and Tchanturia (2015) evaluated the effects of eight studies, indicating an overall within-group effect size between pre and post-treatment assessment of Hedge's $g=$ 0.79, $95 \%$ Confidence Interval (CI) $[0.44,1.12]$, and $g=1.32,95 \%$ CI $[1.02,1.64]$, for two of the most frequently used outcome measures, the Frost Multidimensional Perfectionism Scale (FMPS; Frost, Marten, Lahart, \& Rosenblate, 1990); subscales Concern over Mistakes and Personal Standards. Likewise, the average within-group effect sizes for self-report measures of anxiety, $g=0.52,95 \%$ CI [0.23, 0.81], and depression, $g=0.64,95 \%$ CI [0.35, 0.92], also suggest benefits for those patients receiving treatment and lending some support for the transdiagnostic perspective of clinical perfectionism.

However, access to the right type of care is often limited, making other treatment formats important to consider. Internet-Based Cognitive Behavior Therapy (ICBT) has been shown to be useful for many psychiatric disorders (Andersson, 2016), e.g., panic disorder, social anxiety disorder, and major depressive disorder. In terms of clinical perfectionism, investigations by Arpin-Cribbie, Irvine, and Ritvo (2012), Radhu et al. (2012) and Egan, van Noort, et al. (2014) indicated that ICBT can be beneficial, both for clinical perfectionism and depression and anxiety. However, these studies included relatively small samples and did not involve any guidance from a therapist. As most research on ICBT suggests that the benefits of treatment are greater with support (Andersson, Cuijpers, Carlbring, Riper, \& Hedman, 2014), i.e., larger effect sizes and fewer dropouts, this is an issue that also warrants further investigation in relation to clinical perfectionism. The aim of the current study was therefore to investigate the efficacy of guided ICBT in a large-scale clinical trial for treatment-seeking individuals from the general population. In addition, because no prior investigation of predictors of treatment outcome has been performed, an additional purpose was to explore if there were any variables that might be associated with benefitting from treatment. 


\section{Method}

\section{Participants}

Patients were self-recruited through advertisements in the Swedish media and social media platforms. Individuals interested in participating visited a website created for the purpose of the current study in order to complete a screening process and submit a written informed consent form (www.iterapi.se/sites/devin). Inclusion criteria were fluency in Swedish as assessed during a clinical interview via telephone, minimum 18 years of age, and perfectionism as the primary concern. Cutoff on perfectionism scores was not used as an inclusion criterion. Exclusion criteria were ongoing psychological treatment or counseling, any change in psychotropic medication twelve weeks prior to entering treatment, pregnancy, other conditions warranting more intensive care, e.g., psychosis, anorexia nervosa, and suicidal ideation, as determined using the MINI-International Neuropsychiatric Interview (Sheehan et al., 1998). Other psychiatric disorders were allowed as long as clinical perfectionism was deemed the principal problem.

\section{Procedure}

Individuals having registered their interest to participate completed an online screening process consisting of sociodemographic and self-report measures. In total, 163 fulfilled the inclusion criteria and were called up via telephone for a clinical interview using the MINI. All interviewed cases were then reviewed during a case management conference by the principal investigator (GA), who made the final decision about inclusion and was also clinically responsible for all patients in the study. In turn, 156 were deemed eligible for inclusion and randomized into one of two conditions; treatment or wait-list control. Randomization was performed by an individual external to the current study through a random numbers generator 


\title{
ICBT FOR PERFECTIONISM
}

(www.random.org), according to a 1:1 ratio. Six individuals chose not to participate after the random allocation, but are included in the Intention-To-Treat (ITT) sample. The two conditions were analyzed on all primary and secondary outcome measures at pre-treatment assessment and their sociodemographics to check for any potential deviation from randomness. Those assigned to wait-list control were more likely to have had, or be on, psychotropic medication, $\chi^{2}(1)=4.85$, and to have had prior psychological treatment or counseling, $\chi^{2}(1)=4.11$. A flow chart can be obtained in Figure 1, and the descriptive statistics of the patients in Table 1 .

All communication between the therapists and patients, distribution of modules, and administration of self-report measures at pre- and post-treatment assessment were conducted through a secure online platform connected to the website of the current study. In order to log on, the patients had to use an auto generated identification code generated during the screening process, e.g., 1234abcd, a strong personal password, and a six-letter code sent via SMS. This type of electronic identification, i.e., SSL certificate, and two-step verification is similar to the systems used by many governmental agencies, ensuring anonymity and safety throughout the treatment period (Vlaescu, Alasjö, Miloff, Carlbring, \& Andersson, In press). Furthermore, because the patients completed the self-report measures directly online using their computer, the risk for data distortion or data loss was minimized. Ethics approval was received from the Regional Ethical Board in Linköping, Sweden (Dnr: 2015/419-31).

\author{
(INSERT FIGURE 1)
}

(INSERT TABLE 1)

\section{Measures}




\section{ICBT FOR PERFECTIONISM}

\section{Primary Outcome Measures}

A self-report measure of perfectionism was administered to the patients, the FMPS. Two of the subscales, Concern over Mistakes and Personal Standards, were used as the primary outcome measures. The first is comprised of nine items associated with worries over making mistakes, and the second consists of seven items that reflect setting high standards of performance, both being scored on a five-point scale, from "Strongly disagree" (1) to "Strongly agree" (5), range in scores 9-45 and 7-35, respectively. The two subscales have excellent internal consistency, Cronbach's $\alpha=.88$ and .83 ; in the current study Concern over Mistakes $=.85$ and Personal Standards $=.65$, and correlates well with other self-report measures of perfectionism (Frost et al., 1990).

\section{Secondary Outcome Measures}

The remaining subscales of the FMPS, i.e., Doubts about Action, Parental Expectations, Parental Criticism, and Organization, investigate supplementary factors related to perfectionism, and is scored the same way as FMPS Concern over Mistakes and Personal Standards. The number of items in each subscale varies between 4-6, and demonstrate adequate to excellent internal consistency, .77-.93 (Frost et al., 1990); in the current study Doubts about Action $=.52$, Parental Expectations $=.90$, Parental Criticism $=.86$, and Organizaion $=.83$. Range in scores; Doubts about Action 4-20, Parental Expectations 5-25, Parental Criticism 4-20, and Organizaion 6-30.

The twelve-item Clinical Perfectionism Questionnaire (CPQ; Stoeber \& Damian, 2014) examines the level of clinical perfectionism and is scored on a four-point scale, $0-4$, from "Not at all" to "All of the time", range in scores 0-48. The CPQ has adequate internal consistency, 73 (the current study .66), comprising the two factors perfectionistic strivings and concerns. The nine-item Patient Health Questionnaire (PHQ-9) assesses the degree of 


\section{ICBT FOR PERFECTIONISM}

depression and is scored on a four-point scale, from "Not at all" (0) to "Nearly every day" (4), range in scores 0-36. The PHQ-9 has excellent internal consistency, .89 (Löwe, Kroenke, Herzog, \& Gräfe, 2004); in the current study .84. The seven-item Generalized Anxiety Disorder (GAD-7) determines the level of anxiety and worry and is scored on a four-point scale from "Not at all" (0) to "Nearly every day" (4), range in scores $0-28$. The GAD-7 has excellent internal consistency, .92 (Dear et al., 2011); in the current study .87. The forty-item Dysfunctional Attitude Scale (DAS; Weissman \& Beck, 1978) investigates maladaptive beliefs often found to be related to self-criticism and is scored on a seven-point scale from "Strongly disagree" (1) to "Strongly agree" (7), range in scores 40-280. The DAS has an excellent internal consistency, .93; in the current study .91. The twelve-item Brunnsviken Brief Quality of Life Scale (BBQ; Lindner et al., 2015) explores the level of quality of life based on six different areas, e.g., leisure, and level of importance, e.g., "my leisure time is important to me". The BBQ is scored on a four-point scale from "Strongly disagree" (1) to "Strongly agree" (4), range in scores 0-96. The BBQ has an adequate internal consistency, .76, the current study .70. The twelve-item Self-Compassion Scale - Short Form (SCS-SF) examines the degree of self-compassion and is scored on a five-point scale from "Almost never" (1) to "Almost all of the time" (5), range in scores 5-60. The SCS-SF has a good internal consistency, .86 (Raes, Pommier, Neff, \& Van Gucht, 2011); in the current study .79.

\section{Treatment and Therapists}

The treatment program in the current study was based on a protocol for clinical perfectionism, developed by Egan, Wade, Shafran, and Antony (2014), which was translated and adapted to a Swedish context. It includes psychoeducation regarding CBT, clinical perfectionism, and the cognitive-behavioral conceptualization of maintaining factors, as well as information and a number of exercises, such as, cost-benefit analyses, value clarification, 


\section{ICBT FOR PERFECTIONISM}

behavioral experiments, identifying maladaptive beliefs, using graded exposure, overcoming procrastination, implementing problem-solving techniques, relapse prevention, and selfcompassion. In comparison to the original 8 session protocol (see Egan, van Noort et al., 2014), however, some additional changes were made with regard to the content; a new order of the modules was introduced and a greater emphasis was put on behavioral interventions. The modules were as follows: 1) Understanding your perfectionism, 2) Your own model, values, and motivation, 3) Surveys and experiments, 4) Dealing with perfectionistic behaviors, 5) New ways of thinking, 6) Self-criticism or self-compassion, 7) Self-worth, and 8) Maintain and continue positive change. The eight modules were delivered weekly to the patients on the secure online platform, with a treatment period of eight weeks in total. Each module consisted of an average of 12 pages of text, graphical material, videos and exercises, totaling 121 A4-pages. Patients receiving guided ICBT were advised to complete one module per week, each being released on Mondays regardless of treatment progress, i.e., the same pace for everyone. The exercises were to be completed and sent to their respective therapist on Thursdays, while homework assignments were handed in on Sundays. The therapists provided feedback within 24 hours on each of these occasions. The therapists were six master's degree-level students in clinical psychology, all having completed one and a half years of basic clinical training in CBT. The patients were assigned to their respective therapist through randomization, and the dedicated time per patient and week was on average 17 minutes. In order to ensure treatment integrity and discuss potential problems, two of the study supervisors provided one hour of clinical supervision weekly (AR) and biweekly (RS). For the patients on wait-list control, no modules or therapist contact was available, however, the full treatment was obtained after the first treatment period had ended.

\section{Statistical Analysis}




\section{ICBT FOR PERFECTIONISM}

A priori power was calculated with $\mathrm{G}^{*}$ Power, using a between-group effect size of Cohen's $d=1.00$ as the estimated effect for the comparison between the treatment and control conditions at post-treatment assessment. This was based on a review by (Lloyd et al., 2015), however, because it did not contain information of between-group effect sizes, these were calculated using the results on FMPS Concern over Mistakes and Personal Standards from the clinical trials that included wait-list control, range $d=0.19-1.14,95 \%$ CI [-0.63-2.04] $((k=$ 3 ). With a power of 0.95 and $\alpha$ of .05 , a total of 54 patients divided into two conditions would suffice. Sociodemographics and symptom severity for the two conditions at pre-treatment assessment were explored using two-sided independent $t$-tests and Pearson $\chi^{2}$-tests. Similarly, dropout, defined as those patients not completing the post-treatment assessment, were compared to those completing it, regardless of the number of modules that were finished.

To account for missing data, Multiple Imputation (MI) using Bayesian analysis was performed in Mplus (Muthén \& Muthén, 1999). The imputation procedure utilized all of the primary and secondary outcome measures as well as the number of modules completed. Analysis of Covariance (ANCOVA) was then performed for all of the self-report measures to investigate the treatment results at post-treatment assessment, i.e., comparing the two conditions with regard to change over time, using the dependent variables as covariates to adjust for scores at pre-treatment assessment as the covariates. Ten imputed data sets were specified and the parameter estimates for the ANCOVA were averaged over the set of ten analyses. The presentations of the results are divided by ITT and Completer analysis. Using the results from the ANCOVA, wWithin-group effect sizes were assessed using from the difference in means between the two measurement points for each condition, divided by the pooled standard deviation, while the between-group effect sizes utilized the difference in means between the conditions at post-treatment assessment. In line with the recommendations by (Cumming, 2014), 95\% CI was also calculated for the effect sizes using the Campbell 


\section{ICBT FOR PERFECTIONISM}

Collaboration effect size calculator (https://www.campbellcollaboration.org/effect-size-

calculato.html), which indicates if an effect size was statistically significant or not. In addition, clinically significant change was also used in order to determine the number of patients moving outside the dysfunctional range as a consequence of undergoing treatment. Clinically significant change was defined as having a score on the FMPS Concern over Mistakes at posttreatment assessment that was within one standard deviation (6.39) of the mean in the general population (22.32) i.e., $<29$ (Suddarth \& Slaney, 2001). A Reliable Change Index was also computed to show that the difference was considered reliable and not due to measurement error, requiring change scores to exceed 1.96 times the standard error of the measurement (Evans, Margison, \& Barkham, 1998), in the current study 6.79. Deterioration was determined using a negative change score exceeding the Reliable Change Index, as recommended by Rozental et al. (2014).

Moreover, multiple linear regression was performed in Mplus to investigate predictors of treatment outcome, using the score at post-treatment assessment on the FMPS Concern over Mistakes and Personal Standards as the dependent variables, respectively. All predictors were put into the model at the same time via forced entry, adjusting for their scores at pretreatment assessment. Predictors were selected a priori based on prior empirical evidence or theory; age, gender, and clinical severity at pre-treatment assessment as determined by the PHQ-9 and the GAD-7. For instance, being younger, male, and having more comorbid conditions have been shown to be related to increased odds of dropping out from ICBT (Karyotaki et al., 2015), less benefit during the treatment period (Waller \& Gilbody, 2009), and increased odds of deterioration (Rozental, Magnusson, Boettcher, Andersson, \& Carlbring, 2017). Variables that do not cross zero on the 95\% CIs were regarded as significant predictors of treatment outcome, i.e., $p<.05$. However, given the lack of research on 


\section{ICBT FOR PERFECTIONISM}

predictors of treatment outcome related to clinical perfectionism, these results should at best be seen as tentative, warranting further research.

\section{Results}

\section{Attrition and adherence}

Dropout was defined as those patients not completing the post-treatment assessment regardless of the number of treatment modules having been completed. Missing data was thus explored for all of the primary and secondary outcome measures, indicating that $12.2-14.1 \%$ were regarded as dropouts, depending on how many self-report measures were completed by the patient before choosing to end the post treatment assessment. Independent samples $t$-tests did not reveal a difference in terms of completion, except for the FMPS Organization, suggesting that those who completed the post-treatment assessment scored, on average, 1.98 points higher at the pre-treatment assessment, 95\% CI [0.08, 3.88]. Of the sociodemographic variables, only those patients with children were more likely to complete the outcome measures, $\chi^{2}(2)=6.30$. In terms of adherence, a complete overview of the number of patients completing the modules can be found in Table 5.

\section{(INSERT TABLE 5)}

\section{Treatment results}

The ITT analysis of the ANCOVA revealed significanta main effect of condition between-group effect sizes when comparing the conditions at post-treatment assessment, i.e., treatment vs. wait-list control, on all of the primary and secondary outcome measures, except for FMPS Parental Expectations and Parental Criticism. In other words, there was a difference in treatment outcome between the conditions at post-treatment assessment, with patients in 


\section{ICBT FOR PERFECTIONISM}

treatment benefitting more than those in wait-list control on almost all of the primary and secondary outcome measures. The completer analysis obtained similar results, again with no difference for FMPS Parental Expectations and Parental Criticism. The descriptive statistics for the primary and secondary outcome measures for the respective conditions can be obtained in Table 2, and the within-group and between-group effect sizes with their respective 95\% CI can be located in Table 3.

(INSERT TABLE 2)

(INSERT TABLE 3)

\section{Improvement and negative effects}

Improvement, that is, achieving both clinically significant change and exceeding the Reliable Change Index on the FMPS Concern over Mistakes, was attained by 35 (44.9\%) of the patients receiving treatment, as compared to $9(11.5 \%)$ in wait-list control. Patients in the treatment condition had higher odds for improvement compared to those in the control condition, Odds Ratio $=6.24,95 \%$ CI $[2.73,14.25]$. In terms of psychiatric disorders assessed by the MINI (Sheehan et al., 1998), 59 patients receiving treatment and 69 patients in the wait-list control were available for an interview at post treatment assessment. Of those patients in the treatment condition, only $11.9 \%$ fulfilled the criteria for major depressive disorder and/or an anxiety disorder, as compared to $42.0 \%$ in the control condition. These rates were $45.8 \%$ and $55.1 \%$ prior to the treatment period, respectively. Furthermore, deterioration on the FMPS Concern over Mistakes was attained by $3(3.8 \%)$ of the patients receiving treatment, as determined using the Reliable Change Index, in comparison to 4 $(5.1 \%)$ in wait-list control. 
ICBT FOR PERFECTIONISM

\section{Predictors of treatment outcome}

Predictors of treatment outcome were examined, however, none of the predictors suggested a relationship with the scores for FMPS Concern over Mistakes and Personal Standards at post-treatment assessment, each multiple regression analysis having $\mathrm{R}^{2}=.17$. The result for each predictor can be obtained in Table 4 .

(INSERT TABLE 4)

\section{Discussion}

The current study investigated the efficacy of guided ICBT for clinical perfectionism administered as eight modules over eight weeks. The results revealed large within-group effect sizes for the treatment condition on the two primary outcome measures (ITT); FMPS Concern over Mistakes and Personal Standards, $d=0.93-1.03,95 \%$ CI [0.59-1.36]. In addition, the between-group effect sizes, when comparing guided ICBT to wait-list control, were moderate to large, $d=0.68-1.00,95 \%$ CI [0.36-1.33]. Similarly, small to large betweengroup effect sizes were also evident for most of the secondary outcome measures (see Table 3 for an overview). This is comparable to previous studies of CBT for clinical perfectionism, with within-group effect sizes ranging between $g=0.79$ and $1.32,95 \%$ CI [0.44-1.64] for the two subscales of the FMPS. In terms of between-group effect sizes, the clinical trial by ArpinCribbie et al. (2012) revealed similar results, $d=1.00$ for the FMPS Concern over Mistakes, also using wait-list control as the comparator. The findings from the current study thus seem to be analogous to other examples of CBT and guided ICBT for clinical perfectionism. In terms of the number of patients achieving clinically significant change, no such estimates have been provided previously for clinical perfectionism, while in the current study 35 


\section{ICBT FOR PERFECTIONISM}

$(44.9 \%)$ of the patients receiving treatment were improved at post-treatment assessment and only $9(11.5 \%)$ in wait-list control. If compared to other examples of ICBT, albeit for psychiatric disorders, these results are comparable, for instance, $46 \%$ for mixed anxiety disorders (Nordgren et al., 2014). Also, in comparison to face-to-face CBT, with average response rates varying from $43.3 \%$ to $52.7 \%$ depending on the anxiety disorder being treated (Loerinc et al., 2015), these results are alike.

The results suggest that guided ICBT can be beneficial for those patients who experience clinical perfectionism as their primary concern. This is in line with the review by Lloyd et al. (2015), providing evidence for the overall efficacy of treatment interventions for this type of condition, and the findings by Arpin-Cribbie et al. (2012), Egan, van Noort, et al. (2014), and Radhu et al. (2012) indicating that it may also be delivered successfully via the Internet. Furthermore, because of the close relationship between clinical perfectionism and many psychiatric disorders, it is possible that this type of treatment also could be used in a transdiagnostic approach, as proposed by Egan et al. (2011), or in tailored ICBT (Carlbring et al., 2011). The current study did find moderate between-group effect sizes for depression and anxiety, and large for quality of life, giving some credence to this idea, although more research is warranted to assess this more directly. In terms of the secondary outcome measures that did not reveal any differences, i.e., FMPS Parental Expectations and Parental Criticism, it is conceivable that these subscales concern aspects of perfectionism that are less susceptible to change during treatment as they measure perfectionism expressed by the parents. It is also possible that FMPS Concern over Mistakes and Personal Standards capture the key symptoms associated with perfectionism, while the others are more related to its causes, as suggested by Egan et al. (2016), which would explain why these remained largely unaffected. As for the investigation of predictors of treatment outcome, the results were less informative, implying that none of the variables selected a priori had a relationship with 


\section{ICBT FOR PERFECTIONISM}

benefitting from treatment. However, it might be the case that some variables were underpowered to detect meaningful differences, particularly in terms of gender, with $82.1 \%$ of the patients in the treatment group being female. Also, one of the variables was close to revealing an association, namely the PHQ-9 for the FMPS Concern over Mistakes. Given its quite large effect, $\beta=0.25,95 \%$ CI [-0.00, 0.63], this warrants further investigation in the future as it might suggest an important relationship between the severity level of depression and treatment outcome with regard to clinical perfectionism. Further research should thus be conducted to see if the results of the current study can be replicated, but, more importantly, to explore other variables as possible predictors and moderators of treatment outcome.

A number of limitations are important to address when reviewing the results. First, because the FMPS Concern over Mistakes lacks validated cutoffs for differentiating clinical from non-clinical distributions, the population mean provided by Suddarth and Slaney (2001) was used in order to determine clinically significant change, i.e., $<29$. The average level of distress on this subscale was above this endpoint at pre-treatment assessment, $M=34.28$ ( $S D$ $=6.33$ ), however, $18.6 \%$ of patients scored below this limit, suggesting that the severity of their perfectionism might not have been particularly high from the beginning. The issue of clinically significant change is also relevant for the assessment of predictors of treatment outcome, which in the current study was based solely on the scores for the FMPS Concern over Mistakes and Personal Standards at the post treatment assessment. Hence, the clinical significance of the results from the multiple linear regression analysis might be less clear, and an alternative method would have been to use a dummy coded variable representing those patients actually achieving clinically significant change. However, again, without clearly defined cutoffs on the FMPS as for when this has been achieved, such approach is less useful and might also become underpowered. Second, although self-report measures of perfectionism are often distributed in clinical trials as a method for evaluating their efficacy, 


\section{ICBT FOR PERFECTIONISM}

the association with actual behavior is less clear. There is some experimental evidence of higher levels of perfectionism affecting performance (c.f., Slade, Coppel, \& Townes, 2009), but the findings are somewhat mixed and the ecological validity uncertain (Stoeber, 2011). Third, the patients in the current study were highly educated, $76.9 \%$ having at least a university degree, and an absolute majority were women, $86.5 \%$. This is higher than other clinical trials of ICBT, which, in turn, have been criticized for only attracting a certain sociodemographic group (Arnberg, Linton, Hultcrantz, Heintz, \& Jonsson, 2014). However, this is similar to treatment-seeking individuals in general, who tend to be women and have a higher educational level (Vessey \& Howard, 1993). Furthermore, it is also possible that this group identify themselves as perfectionists, or, experience problems with perfectionism, to a greater degree, explaining this skewness in recruitment. Fourth, a major limitation of the current study is the lack of a follow-up assessment, making it impossible to explore the longterm results of the treatment. Hence, although the results were encouraging at post-treatment assessment, more research has to be performed to affirm what benefits guided ICBT can have for clinical perfectionism over a longer time period. Similarly, what aspects contributed to its efficacy needs to be investigated more closely, for instance, if unguided treatment interventions can yield comparable results, as has been shown in the review by Lloyd et al. (2015), or if there are particular aspects of content that are most important.

\section{Conclusion}

Guided ICBT can be an efficient way of alleviating difficulties related to clinical perfectionism. More research is, however, warranted to determine its efficacy in the long-run, and to investigate other variables as potential predictors of treatment outcome.

\section{Acknowledgements}




\section{ICBT FOR PERFECTIONISM}

The authors of the current study would like to acknowledge Linköping University and a Professor's grant to the principal investigator (GA), which made the completion of the clinical trial possible. This research was supported by the National Institute for Health Research Biomedical Research Centre at Great Ormond Street Hospital for Children NHS Foundation Trust and University College London. Furthermore, George Vlaescu is thanked for his excellent webmaster service, and Chloe Yu Shu for providing the original website for the current study. 
ICBT FOR PERFECTIONISM

\section{References}

Andersson, G. (2016). Internet-Delivered Psychological Treatments. Annu Rev Clin Psychol, 12, 157-179. doi:10.1146/annurev-clinpsy-021815-093006

Andersson, G., Cuijpers, P., Carlbring, P., Riper, H., \& Hedman, E. (2014). Guided Internet based vs. face - to - face cognitive behavior therapy for psychiatric and somatic disorders: a systematic review and meta - analysis. World Psychiatry, 13(3), 288-295.

Arnberg, F. K., Linton, S. J., Hultcrantz, M., Heintz, E., \& Jonsson, U. (2014). InternetDelivered Psychological Treatments for Mood and Anxiety Disorders: A Systematic Review of Their Efficacy, Safety, and Cost-Effectiveness. Plos One, 9(5), e98118. doi:10.1371/journal.pone.0098118

Arpin-Cribbie, C., Irvine, J., \& Ritvo, P. (2012). Web-based cognitive-behavioral therapy for perfectionism: a randomized controlled trial. Psychotherapy Research, 22(2), 194-207.

Burns, D. D. (1980). The perfectionist's script for self-defeat. Psychology today, 14(6), 34-52. Carlbring, P., Maurin, L., Törngren, C., Linna, E., Eriksson, T., Sparthan, E., . . Andersson, G. (2011). Individually-tailored, Internet-based treatment for anxiety disorders: A randomized controlled trial. Behaviour Research and Therapy, 49(1), 18-24.

Chang, E. C., Watkins, A., \& Banks, K. H. (2004). How Adaptive and Maladaptive Perfectionism Relate to Positive and Negative Psychological Functioning: Testing a Stress-Mediation Model in Black and White Female College Students. Journal of Counseling Psychology, 51(1), 93.

Cumming, G. (2014). The New Statistics. Psychological science, 25(1), 7-29. doi:doi:10.1177/0956797613504966

Dear, B. F., Titov, N., Sunderland, M., McMillan, D., Anderson, T., Lorian, C., \& Robinson, E. (2011). Psychometric Comparison of the Generalized Anxiety Disorder Scale-7 and the Penn State Worry Questionnaire for Measuring Response during Treatment of 


\section{ICBT FOR PERFECTIONISM}

Generalised Anxiety Disorder. Cognitive Behaviour Therapy, 40(3), 216-227. doi:10.1080/16506073.2011.582138

Egan, S. J., \& Hine, P. (2008). Cognitive behavioural treatment of perfectionism: A single case experimental design series. Behaviour Change, 25(04), 245-258.

Egan, S. J., Shafran, R., Lee, M., Fairburn, C. G., Cooper, Z., Doll, H. A., . . Watson, H. J. (2016). The reliability and validity of the clinical perfectionism questionnaire in eating disorder and community samples. Behavioural and Cognitive Psychotherapy, 44(01), 79-91.

Egan, S. J., van Noort, E., Chee, A., Kane, R. T., Hoiles, K. J., Shafran, R., \& Wade, T. D. (2014). A randomised controlled trial of face to face versus pure online self-help cognitive behavioural treatment for perfectionism. Behaviour Research and Therapy, 63, 107-113.

Egan, S. J., Wade, T. D., \& Shafran, R. (2011). Perfectionism as a transdiagnostic process: A clinical review. Clinical Psychology Review, 31(2), 203-212.

Egan, S. J., Wade, T. D., Shafran, R., \& Antony, M. M. (2014). Cognitive-behavioral treatment of perfectionism. NY: Guilford.

Evans, C., Margison, F., \& Barkham, M. (1998). The contribution of reliable and clinically significant change methods to evidence-based mental health. Evidence-Based Mental Health, 1(3), 70-72. doi:10.1136/ebmh.1.3.70

Frost, R. O., Marten, P., Lahart, C., \& Rosenblate, R. (1990). The dimensions of perfectionism. Cognitive Therapy and Research, 14(5), 449-468.

Handley, A. K., Egan, S. J., Kane, R. T., \& Rees, C. S. (2015). A randomised controlled trial of group cognitive behavioural therapy for perfectionism. Behaviour Research and Therapy, 68, 37-47. 


\section{ICBT FOR PERFECTIONISM}

Karyotaki, E., Kleiboer, A., Smit, F., Turner, D. T., Pastor, A., Andersson, G., . . Carlbring, P. (2015). Predictors of treatment dropout in self-guided web-based interventions for depression: an 'individual patient data'meta-analysis. Psychological medicine, 45(13), $2717-2726$.

Lindner, P., Frykheden, O., Forsström, D., Andersson, E., Ljótsson, B., Hedman, E., . . . Carlbring, P. (2015). The Brunnsviken Brief Quality of life scale (BBQ): Development and psychometric evaluation. Manuscript submitted for publication.

Lloyd, S., Schmidt, U., Khondoker, M., \& Tchanturia, K. (2015). Can psychological interventions reduce perfectionism? A systematic review and meta-analysis. Behavioural and Cognitive Psychotherapy, 43(06), 705-731.

Loerinc, A. G., Meuret, A. E., Twohig, M. P., Rosenfield, D., Bluett, E. J., \& Craske, M. G. (2015). Response rates for CBT for anxiety disorders: need for standardized criteria. Clinical psychology review, 42, 72-82.

Löwe, B., Kroenke, K., Herzog, W., \& Gräfe, K. (2004). Measuring depression outcome with a brief self-report instrument: sensitivity to change of the Patient Health Questionnaire (PHQ-9). Journal of Affective Disorders, 81(1), 61-66.

Muthén, L. K., \& Muthén, B. O. (1999). Mplus User's Guide. Los Angeles, CA: Muthén \& Muthén.

Nordgren, L. B., Hedman, E., Etienne, J., Bodin, J., Kadowaki, Å., Eriksson, S., . . . Carlbring, P. (2014). Effectiveness and cost-effectiveness of individually tailored Internet-delivered cognitive behavior therapy for anxiety disorders in a primary care population: A randomized controlled trial. Behaviour Research and Therapy, 59, 1-11.

Radhu, N., Daskalakis, Z. J., Arpin-Cribbie, C. A., Irvine, J., \& Ritvo, P. (2012). Evaluating a web-based cognitive-behavioral therapy for maladaptive perfectionism in university students. Journal of American College Health, 60(5), 357-366. 


\section{ICBT FOR PERFECTIONISM}

Raes, F., Pommier, E., Neff, K. D., \& Van Gucht, D. (2011). Construction and factorial validation of a short form of the self - compassion scale. Clinical Psychology \& Psychotherapy, 18(3), 250-255.

Rozental, A., Andersson, G., Boettcher, J., Ebert, D. D., Cuijpers, P., Knaevelsrud, C., . . Carlbring, P. (2014). Consensus statement on defining and measuring negative effects of Internet interventions. Internet Interventions, 1(1), 12-19. doi:10.1016/j.invent.2014.02.001

Rozental, A., Magnusson, K., Boettcher, J., Andersson, G., \& Carlbring, P. (2017). For better or worse: An individual patient data meta-analysis of deterioration among participants receiving Internet-based cognitive behavior therapy. J Consult Clin Psychol, 85(2), 160-177. doi:10.1037/ccp0000158

Shafran, R., Cooper, Z., \& Fairburn, C. G. (2002). Clinical perfectionism: A cognitivebehavioural analysis. Behaviour Research and Therapy, 40(7), 773-791.

Shafran, R., \& Mansell, W. (2001). Perfectionism and psychopathology: A review of research and treatment. Clinical Psychology Review, 21(6), 879-906.

Sheehan, D., Lecrubier, Y., Sheehan, K. H., Sheehan, K., Amorim, P., Janavs, J., . . Dunbar, G. (1998). Diagnostic Psychiatric Interview for DSM-IV and ICD-10. The Journal of Clinical Psychiatry, 59, 22-33.

Slade, P. D., Coppel, D. B., \& Townes, B. D. (2009). Neurocognitive correlates of positive and negative perfectionism. International Journal of Neuroscience, 119(10), 17411754.

Steele, A. L., \& Wade, T. D. (2008). A randomised trial investigating guided self-help to reduce perfectionism and its impact on bulimia nervosa: A pilot study. Behaviour Research and Therapy, 46(12), 1316-1323. 


\section{ICBT FOR PERFECTIONISM}

Stoeber, J. (2011). Perfectionism, efficiency, and response bias in proof-reading performance: Extension and replication. Personality and Individual Differences, 50(3), 426-429.

Stoeber, J., \& Damian, L. E. (2014). The Clinical Perfectionism Questionnaire: Further evidence for two factors capturing perfectionistic strivings and concerns. Personality and Individual Differences, 61, 38-42.

Stoeber, J., \& Otto, K. (2006). Positive conceptions of perfectionism: Approaches, evidence, challenges. Personality and Social Psychology Review, 10(4), 295-319.

Stumpf, H., \& Parker, W. D. (2000). A hierarchical structural analysis of perfectionism and its relation to other personality characteristics. Personality and Individual Differences, 28(5), 837-852.

Suddarth, B. H., \& Slaney, R. B. (2001). An investigation of the dimensions of perfectionism in college students. Measurement and Evaluation in Counseling and development, 34(3), 157.

Vessey, J. T., \& Howard, K. I. (1993). Who seeks psychotherapy? Psychotherapy: Theory, Research, Practice, Training, 30(4), 546-553. doi:10.1037/0033-3204.30.4.546

Vlaescu, G., Alasjö, A., Miloff, A., Carlbring, P., \& Andersson, G. (In press). Features and functionality of the Iterapi platform for internet-based psychological treatment. Internet Interventions.

Waller, R., \& Gilbody, S. (2009). Barriers to the uptake of computerized cognitive behavioural therapy: a systematic review of the quantitative and qualitative evidence. Psychological medicine, 39(05), 705-712.

Weissman, A. N., \& Beck, A. T. (1978). Development and validation of the Dysfunctional Attitude Scale: A preliminary investigation. 